\title{
Long-chain $n-3$ DHA reduces the extent of skeletal muscle fatigue in the rat in vivo hindlimb model
}

\author{
Gregory E. Peoples ${ }^{1 *}$ and Peter L. McLennan ${ }^{2}$ \\ ${ }^{1}$ School of Health Sciences and Smart Foods Centre, University of Wollongong, Wollongong, 2522 NSW, Australia \\ ${ }^{2}$ Graduate School of Medicine, University of Wollongong, Wollongong, 2522 NSW, Australia \\ (Submitted 19 December 2012 - Final revision received 15 August 2013 - Accepted 20 August 2013 - First published online 11 November 2013)
}

\begin{abstract}
Dietary fish oil modifies skeletal muscle membrane fatty acid composition and oxygen efficiency similar to changes in the myocardium. Oxygen efficiency is a key determinant of sustained force in mammalian skeletal muscle. Therefore, in the present study, we tested the effects of a fish-oil diet on skeletal muscle fatigue under the stress of contraction using the rat in vivo autologous perfused hindlimb model. For 8 weeks, male Wistar rats were fed a diet rich in saturated fat (SF), a diet rich in $n-6$ PUFA or a diet rich in long-chain (LC) $n$-3 PUFA DHA derived from fish oil. In anaesthetised, mechanically ventilated rats, with their hindlimbs perfused with arterial blood at a constant flow, the gastrocnemius-plantaris-soleus muscle bundle was stimulated via sciatic nerve $(2 \mathrm{~Hz}, 6-12 \mathrm{~V}, 0.05 \mathrm{~ms})$ to contract repetitively for $30 \mathrm{~min}$. Rats fed the $n$-3 PUFA diet developed higher maximum twitch tension than those fed the SF and $n$ - 6 PUFA diets $(P<0.05)$ and sustained twitch tension through more repetitions before the tension declined to $50 \%$ of the maximum twitch tension $(P<0 \cdot 05)$. The $n-3$ PUFA group used less oxygen for tension developed and produced higher venous lactate concentrations with no difference in glycogen utilisation compared with the SF and $n-6$ PUFA groups. These results further support that incorporation of DHA into skeletal muscle membranes increases the efficiency of oxygen use over a range of contractile force and this is expressed as a higher sustained force and prolonged time to fatigue.
\end{abstract}

Key words: Fish oil: Oxygen consumption: Fatigue: DHA

Skeletal muscle fatigue, or the decline in expectant force development, is tightly coupled with oxygen supply and demand $^{(1-4)}$. The ability of skeletal muscle to rapidly adjust developed force when oxygen is limiting or demand is increased is well established and associated with reduced muscle function without disturbance in tissue levels of ATP ${ }^{(5)}$. Therefore, efficient use of available oxygen is of upmost importance with regard to repetitive and sustained skeletal muscle force production.

Structural membrane composition directly affects cell function $^{(6)}$. When long-chain (LC) $n-3$ PUFA from dietary fish oil are incorporated into myocardial membranes ${ }^{(7-10)}$, the function of the heart is enhanced, particularly when oxygen supply is compromised ${ }^{(8,11)}$. In skeletal muscle membranes, a similar process of incorporation occurs, whereby LC $n-3$ PUFA DHA concentrations are well above the dietary or circulating concentrations ${ }^{(10,12-14)}$. A number of key metabolic ${ }^{(15)}$ and physiological ${ }^{(16)}$ processes have been reported to be associated with the increased concentrations of LC $n-3$ PUFA DHA in skeletal muscle membranes.
Preliminary evidence has emerged that skeletal muscle oxygen consumption is modulated, as in the heart, after fish oil supplementation. In trained cyclists, dietary fish oil supplementation, delivering high concentrations of DHA, has been shown to reduce heart rate and whole-body oxygen consumption during prolonged submaximal cycling performance ${ }^{(17)}$. Skeletal muscle is the dominant consumer of oxygen during intense exercise, and this implies that the efficiency of oxygen use by the working muscles is enhanced. To directly test this hypothesis, oxygen consumption has been measured across autoperfused contracting hindlimbs ${ }^{(18)}$. Incorporation of DHA into skeletal muscle membranes results in oxygen cost-saving effects, as observed in the heart. However, oxygen consumption has been measured at only one time point in the fatigue process. Muscle fatigue commences from the initiation of force production, and in light of the critical role of oxygen supply and demand, this detailed relationship deserves further investigation.

No clear improvement in human fatigue tolerance has yet been demonstrated with dietary fish oil ${ }^{(19-21)}$. This can be attributed either to varying $n$-3 PUFA doses and durations of

Abbreviations: LC, long chain; SF, saturated fat.

* Corresponding author: Dr G. E. Peoples, fax +6124221 3486, email peoples@uow.edu.au 
supplementation $^{(22)}$ or to fatigue measures with poor reliability and sensitivity. The latter can largely be ascribed to the focus of research on potential cardiac, peripheral vascular or haematological mechanisms for improved exercise endurance. In other words, whole-body exercise tolerance is multifactorial, and unless careful design is employed, the many underlying mechanisms as to why one cannot sustain exercise, including the properties of skeletal muscle itself, cannot be resolved. Specifically, with regard to our oxygen modulation hypothesis, neither human ${ }^{(17)}$ nor animal $^{(14)}$ studies have primarily been designed to directly examine the effects of LC $n-3$ PUFA on muscle endurance, focusing instead on cardiac endurance and on the recovery of skeletal muscle contractile force between repeat contractile bouts. Moreover, a previous study has employed a muscle stimulation rate too slow and of insufficient duration to elicit a major depression in skeletal muscle force production and thus enable an objective measure of muscle fatigue.

To date, no attempt has been made to investigate the direct impact of fish oil supplementation and specifically incorporation of LC $n-3$ DHA into skeletal muscle membranes on prolonged contractile fatigue resistance. Therefore, the aim of the present study was to use the autologous blood-perfused hindlimb method in rats ${ }^{(18,23)}$ to investigate the effects of dietary LC $n$-3 PUFA, and specifically DHA, on skeletal muscle fatigue resistance including measuring oxygen consumption at multiple time points of force depression. It was hypothesised that the increased efficiency of skeletal muscle oxygen consumption, at multiple time points, would be expressed as an attenuation of muscle force depression over a sustained bout of contraction eliciting fatigue.

\section{Experimental methods}

In total, eighteen young male Wistar rats (400-500 g; 18-20 weeks of age) were used for the study and were evenly divided into groups of six per dietary intervention. The rats were housed two per cage in the animal facility of the University of Wollongong with temperature being maintained at $23-25^{\circ} \mathrm{C}$ under a $12 \mathrm{~h}$ light-dark cycle. Experiments were assessed and approved by the Animal Ethics Committee of the University of Wollongong, and all national and institutional guidelines were followed.

\section{Diet composition}

In the present study, three diets (saturated fat (SF), n-6 PUFA and $n-3$ PUFA) were prepared. All the diets contained a balanced mix of macro- and micronutrients to avoid any nutrient deficiencies based on the AIN-93M diet ${ }^{(24)}$. In brief, the diets were designed to deliver (1) similar total PUFA amounts in the $n$ - 6 PUFA $(36.65 \mathrm{~g} / \mathrm{kg})$ and $n$ - 3 PUFA $(31.40 \mathrm{~g} / \mathrm{kg})$ diets, (2) similar $n$-6 PUFA amounts in the $n-3$ PUFA $(5.01 \mathrm{~g} / \mathrm{kg})$ and SF $(4.77 \mathrm{~g} / \mathrm{kg})$ diets and (3) similar $n-3$ PUFA amounts in the $n$-6 PUFA $(0.49 \mathrm{~g} / \mathrm{kg})$ and SF $(0.58 \mathrm{~g} / \mathrm{kg})$ diets. Olive oil was used as a light (refined) oil; thus, the diets were free of confounding sources of natural antioxidants; beef tallow was used as saturated fat; safflower oil as a source of $n$ - 6 PUFA;
high-DHA tuna fish oil as a source of $n-3$ PUFA $(\alpha$-linolenic acid $0.56 \mathrm{~g} / \mathrm{kg}$, EPA $4.87 \mathrm{~g} / \mathrm{kg}$ and DHA $20.2 \mathrm{~g} / \mathrm{kg}$ ) (Nu-Mega Ingredients). All the diets contained $10 \%$ fat by weight. The proportions of fat sources have been reported previously ${ }^{(14)}$ : the SF diet contained 30:70 olive oil and beef tallow; the $n-6$ PUFA diet contained 50:50 olive oil and safflower oil; the $n$-3 PUFA diet contained 30:70 olive oil and tuna oil. The diets were prepared from purified ingredients and stored at $-20^{\circ} \mathrm{C}^{(25)}$.

\section{Study design}

The rats were fed a washout diet containing olive oil for $14 \mathrm{~d}$ to standardise muscle membrane fatty acid composition. The eighteen rats were then randomly allocated to three groups and fed the SF, safflower oil ( $n$-6 PUFA) or tuna fish-oil ( $n$-3 PUFA) diet for 8 weeks (ad libitum). Fresh food was provided twice per week, and daily intake was estimated by weighing the remainder from the previous feeding. The weight of the rats was measured once per week.

\section{Surgical preparation for ventilation, muscle perfusion and stimulation}

The autologous pump-perfused hindlimb method has been described previously in both canines ${ }^{(26)}$ and rats ${ }^{(14,23)}$. In brief, rats (18-20 weeks) were anaesthetised (sodium pentobarbital $6 \mathrm{mg} / 100 \mathrm{~g}$ intraperitoneally) with supplementary injections $(2 \mathrm{mg} / 100 \mathrm{~g}$ intraperitoneally) as required. The experiments were carried with each of the rats and the perfusion system being enclosed in a heated Perspex chamber $\left(32.2 \pm 0.3^{\circ} \mathrm{C}\right)$ with the body temperature of the rats being maintained at $37^{\circ} \mathrm{C}$ (rectal temperature). The specific diet group that each rat belonged to was blinded to the scientist carrying out the procedure. The rats were prepared for artificial ventilation $(1 \mathrm{ml} / 100 \mathrm{~g}$ body weight, Rodent Ventilator 7025; Ugo Basile) and blood pressure recording by intubation of the trachea and cannulation of the left carotid artery, respectively.

All cannulae were fluid filled with saline containing $6 \%$ dextran (w/v) and $5000 \mathrm{IU}$ heparin/100 ml (Sigma-Aldrich). Arterial (oxygenated) blood was accessed via a cannula inserted into the right femoral artery (non-perfused leg) towards the heart and was connected to a resealing flexitube, passed through a peristaltic roller pump (Minipuls 3; Gilson). Arterial blood was then passed through a cannula inserted into the left femoral artery (perfused leg) and positioned to deliver blood directly to muscle groups below the knee. A T-junction inserted in the blood flow line on the perfused hindlimb side of the pump was connected to a pressure transducer (Argon CDXIII; Maxim Medical) for the measurement of hindlimb perfusion pressure. The return of deoxygenated venous blood to the heart and lungs was achieved via passive flow through a cannula inserted into the left femoral vein of the perfused leg and the right jugular vein.

Hindlimb muscle contraction was achieved via sciatic nerve stimulation. A bipolar electrode (F-B5EI; Grass Technologies) was placed under the nerves for direct electrical stimulation 
of the trunk supplying the muscles of the gastrocnemiusplantaris-soleus muscle bundle. The nerve was crushed above the stimulation point to prevent retrograde conduction, standard practice in hindlimb experiments ${ }^{(27)}$. The gastrocnemius-plantaris-soleus muscle group tendons were tied with non-compliant silk and connected to a force transducer (FT03C; Grass Technologies). Saline-soaked gauze was placed over the muscles to prevent drying.

\section{Hindlimb perfusion and stimulation protocol}

The hindlimbs of the rats were perfused for $30 \mathrm{~min}$ at $1 \mathrm{ml} / \mathrm{min}$ with no stimulation to allow for perfusion pressure to reach steady state (approximately $100 \mathrm{mmHg}$ ), and then baseline arterial and venous blood samples $(200 \mu \mathrm{l})$ were drawn. The flow rate was reset and maintained at $2 \mathrm{ml} / \mathrm{min}$ during a single $30 \mathrm{~min}$ repetitive twitch stimulation bout $(2 \mathrm{~Hz}$, $7-12 \mathrm{~V}, 0.05 \mathrm{~ms})$ to the sciatic nerve. Venous samples were collected throughout the stimulation bout period at time points $30 \mathrm{~s}, 60 \mathrm{~s}, 2.5 \mathrm{~min}$ and $5 \mathrm{~min}$ and then every $5 \mathrm{~min}$ until $30 \mathrm{~min}$.

\section{Measurements}

Blood pressure and twitch force. Data were referenced to ground and amplified (Onspot Australia). The data acquisition software (Labview for Windows; National Instruments) was used to collect perfusion pressure and twitch force data throughout the perfusion trials (sampling rate $200 \mathrm{~Hz}$ ).

Twitch characteristics were analysed at time points corresponding to blood sampling. Developed tension and other characteristics were averaged from ten consecutive contractions. Peak tension was defined as the highest developed force in each twitch curve. Maximal peak twitch tension was defined as the mean tension over the ten highest peak tensions. The first derivative of developed tension was used to determine the maximum rate of developed tension and relaxation. The area under the twitch curve (tension-time index) was calculated relative to peak tension at the point of initial contraction (first ten contractions), at the point of maximum contraction (maximal ten contractions) and after $30 \mathrm{~min}$ of contraction (final ten contractions).

Blood samples. Arterial and venous blood samples $(200 \mu \mathrm{l})$ were collected via resealing silicone sections of the cannulae located proximal and distal to the hindlimbs. Of the $200 \mu \mathrm{l}$ blood sampled, $80 \mu \mathrm{l}$ were used for the measurement of $\mathrm{PO}_{2}, \mathrm{PCO}_{2}, \mathrm{Na}^{+}, \mathrm{K}^{+}, \mathrm{pH}$ and $\mathrm{Hb}$ using the laboratory blood gas and electrolyte machine (ABL77; Radiometer). The remaining $120 \mu \mathrm{l}$ were spun down on a benchtop microcentrifuge (Millipore), and the plasma was removed and frozen for later analysis of blood glucose and lactate. Erythrocytes from the plasma samples were resuspended in an equal volume of saline and re-injected into the venous side of the perfusion. $\mathrm{Hb}$ concentrations were maintained above $12.5 \mathrm{~g} / 100 \mathrm{ml}$ whole blood throughout the experiments.

Muscle samples. Control muscle samples (gastrocnemius, plantaris and soleus) were collected from the right (nonperfused) leg immediately before the right femoral artery cannulation to be used as within-animal control tissue. At the completion of each trial and while the blood was still flowing, left leg (perfused and contracted) muscle samples (gastrocnemius, plantaris and soleus) were rapidly extracted. All the muscles were freeze-clamped and stored $\left(-80^{\circ} \mathrm{C}\right)$ for later analysis. Soleus muscle was sampled as red/oxidative tissue and gastrocnemius muscle was sampled as white/ glycolytic tissue. Additional muscle samples (deep posterior tibial, extensor digitorum longus and tibialis anterior) from the perfused hindlimbs were collected to determine the total wet weight of the skeletal muscle involved in the perfusion.

\section{Statistical analysis}

Two-way ANOVA was conducted with diet and time main effects and diet $\times$ time interaction using Statistix, version 8 (Analytical Software). Individual dietary pairwise comparisons were made using a corrected Bonferroni post hoc analysis for multiple comparisons of individual means. $\alpha$ was set at $P<0 \cdot 05$. Data are expressed as means with their standard errors.

\section{Results}

\section{Food consumption and body weight}

There was no significant difference in the quantity of food consumed between the dietary groups at either the start or the end of the trials or in the mean daily intake (SF: 6.68 (0.20 SEM) g/100 g body weight per d; $n$-6 PUFA: 6.54 (0.15 SEM) g/100 g body weight per d; $n$-3 PUFA: 6.37 ( 0.13 SEM) $\mathrm{g} / 100 \mathrm{~g}$ body weight per $\mathrm{d}$ ) over the 8 -week feeding period. The mean body weight of the $n$ - 6 PUFA group (544 (35 SEM)g) was higher than that of the SF (465 (28 SEM)g) and $n$-3 PUFA (472 (34 SEM)g) groups $(P<0.05)$. There were no significant differences in the wet weight of the gastrocnemius-plantaris-soleus muscle bundle, which represented the contracting skeletal muscle bundle, between the dietary groups (SF: 3.08 (0.21 SEM) g wet weight; $n-6$ PUFA: 2.93 $(0 \cdot 17$ SEM)g wet weight; $n-3$ PUFA: 2.51 ( $0 \cdot 23$ SEM)g wet weight).

\section{Baseline perfusion}

Stable baseline conditions were achieved in all the dietary groups (Table 1). Rectal temperature was equally well maintained during all the surgical procedures and experimental conditions in all the dietary groups (SF: $37 \cdot 0 \pm 0 \cdot 9^{\circ} \mathrm{C} ; n-6$ PUFA: $36.7 \pm 0.6^{\circ} \mathrm{C} ; n-3$ PUFA: $\left.36 \cdot 0 \pm 0.7^{\circ} \mathrm{C}\right)$. There was no significant difference in hindlimb perfusion pressure following $30 \mathrm{~min}$ of controlled flow ( $1 \mathrm{ml} / \mathrm{min}$ ) (SF: 109 (11 sEM) $\mathrm{mmHg}$; n-6 PUFA: 102 (3 SEM) mmHg; $n$-3PUFA: 106 (6 SEM) mmHg). In all the dietary groups, artificial ventilation (SF: 151 (12 SEM) $\mathrm{ml} / \mathrm{min}$; $n-6$ PUFA: 172 (13 SEM) $\mathrm{ml} / \mathrm{min}$; $n-3$ PUFA: 147 (11 SEM) $\mathrm{ml} / \mathrm{min}$ ) and resulting $\mathrm{PaO}_{2}$ were sufficient to achieve approximately $98 \%$ oxygen saturation of erythrocytes and corresponding high content of arterial oxygen (approximately $19-20 \mathrm{ml} / 100 \mathrm{ml}$ ). Baseline venous $\mathrm{PO}_{2}$ was significantly 
Table 1. Baseline (non-contracting) arterial and venous blood gas and resulting oxygen consumption following $30 \mathrm{~min}$ of perfusion at a constant flow rate $(1 \mathrm{ml} / \mathrm{min})^{*}$

(Mean values with their standard errors, $n 6$ per group)

\begin{tabular}{|c|c|c|c|c|c|c|}
\hline & \multicolumn{2}{|c|}{ SF } & \multicolumn{2}{|c|}{$n-6$ PUFA } & \multicolumn{2}{|c|}{$n$-3 PUFA } \\
\hline & Mean & SEM & Mean & SEM & Mean & SEM \\
\hline \multicolumn{7}{|l|}{ Arterial } \\
\hline $\mathrm{pH}$ & 7.52 & 0.02 & 7.54 & 0.02 & 7.54 & 0.02 \\
\hline $\mathrm{PaO}_{2}(\mathrm{mmHg})$ & 94 & 3 & 94 & 3 & 98 & 4 \\
\hline $\mathrm{PaCO}_{2}(\mathrm{mmHg})$ & 33 & 2 & 35 & 2 & 33 & 1 \\
\hline $\mathrm{Hb}(\mathrm{g} / 100 \mathrm{ml})$ & $13 \cdot 0$ & 0.5 & $12 \cdot 4$ & 0.5 & $13 \cdot 2$ & 0.3 \\
\hline $\mathrm{CaO}_{2}(\mathrm{ml} / 100 \mathrm{ml})$ & $18 \cdot 3$ & 0.6 & $18 \cdot 0$ & $0 \cdot 6$ & $18 \cdot 6$ & 0.4 \\
\hline $\mathrm{HCO}_{3}^{-}$concentration (mM) & $26 \cdot 95$ & 1.03 & $30 \cdot 01$ & 1.94 & $29 \cdot 0$ & $1 \cdot 38$ \\
\hline Lactate concentration (mM) & 1.74 & $0 \cdot 17$ & 1.64 & 0.14 & 1.71 & 0.09 \\
\hline Glucose concentration (mM) & 5.87 & 0.64 & 5.75 & $0 \cdot 18$ & $6 \cdot 1$ & 0.08 \\
\hline \multicolumn{7}{|l|}{ Venous } \\
\hline $\mathrm{pH}$ & $7 \cdot 45$ & 0.02 & $7 \cdot 44$ & 0.02 & $7 \cdot 42$ & 0.02 \\
\hline $\mathrm{PvO}_{2}(\mathrm{mmHg})$ & 46 & 3 & 47 & 2 & 49 & 2 \\
\hline $\mathrm{PvCO}_{2}(\mathrm{mmHg})$ & 42 & 1 & 45 & 3 & 40 & 2 \\
\hline $\mathrm{Hb}(\mathrm{g} / 100 \mathrm{ml})$ & $13 \cdot 2$ & 0.4 & 12.95 & 0.44 & $13 \cdot 2$ & 0.3 \\
\hline $\mathrm{CvO}_{2}(\mathrm{ml} / 100 \mathrm{ml})$ & $15 \cdot 19$ & 0.76 & $15 \cdot 03$ & 0.36 & 14.96 & 0.51 \\
\hline $\mathrm{HCO}_{3}^{-}$concentration (mM) & $28 \cdot 2$ & $1 \cdot 3$ & $30 \cdot 38$ & $2 \cdot 07$ & $27 \cdot 83$ & 1.69 \\
\hline Lactate concentration (mM) & 1.96 & 0.13 & $2 \cdot 1$ & 0.14 & $2 \cdot 0$ & 0.11 \\
\hline Glucose concentration (mM) & 5.57 & 0.56 & 5.48 & 0.74 & $6 \cdot 0$ & 0.84 \\
\hline \multicolumn{7}{|l|}{ Oxygen consumption } \\
\hline$(a-v) \mathrm{O}_{2}(\mathrm{ml} / 100 \mathrm{ml})$ & $3 \cdot 14$ & 0.51 & 2.95 & 0.52 & 2.96 & 0.23 \\
\hline $\mathrm{VO}_{2}(\mu \mathrm{mol} / \mathrm{g} \times \mathrm{min})$ & 0.43 & 0.07 & 0.42 & 0.06 & 0.45 & 0.06 \\
\hline
\end{tabular}

$\mathrm{SF}$, saturated fat; $\mathrm{PaO}_{2}$, arterial $\mathrm{O}_{2}$ partial pressure; $\mathrm{PaCO}_{2}$, arterial $\mathrm{CO}_{2}$ partial pressure; $\mathrm{CaO}_{2}$, arterial content of $\mathrm{O}_{2} ; \mathrm{HCO}_{3}^{-}$, carbonate concentration; $\mathrm{PvO}_{2}$, venous $\mathrm{O}_{2}$ partial pressure; $\mathrm{PvCO}_{2}$, venous $\mathrm{CO}_{2}$ partial pressure; $\mathrm{CvO}_{2}$, venous content of $\mathrm{O}_{2} ;(a-v) \mathrm{O}_{2}$, arterial venous $\mathrm{O}_{2}$ difference; $\mathrm{VO}_{2}, \mathrm{O}_{2}$ consumption.

* There were no significant differences between the dietary groups for variables measured at baseline $(P>0.05)$.

lower than arterial $\mathrm{PO}_{2}(P<0 \cdot 05)$, demonstrating basal oxygen consumption by the resting muscle bundle. There was no effect of diet on the $(a-v) \mathrm{O}_{2}$ difference or basal oxygen consumption. There were no differences in the basal blood lactate or glucose concentrations between the dietary groups.

\section{Tension development and oxygen consumption}

In all the dietary groups, twitch tension increased from the tension developed in the first twitch to reach maximum twitch tension after about 150 contractions (60-80 s). Peak twitch tension then gradually declined over the course of $30 \mathrm{~min}$ repetitive stimulation bouts (Fig. 1(a); $P<0.05$ ). The $n$-3 PUFA group developed significantly greater maximum twitch tension and attained greater peak tensions throughout the stimulation period $(P<0 \cdot 05)$. There was no difference in time from initial twitch to the twitch of maximum tension (Fig. 2(a)) or in time from the twitch of maximum tension to the twitch of $80 \%$ maximum tension between the dietary groups (Fig. 2(b)). However, in the $n-3$ PUFA group, the peak twitch tension took longer time to decline to $50 \%$ of the maximum compared with that in the SF and n-6 PUFA groups, with the difference being significantly different from that in the $n-6$ PUFA group $(P<0 \cdot 05)$.

Oxygen consumption increased with time during the repetitive stimulation bouts in all the groups $(P<0.05$ for time) and stabilised after about 10 min of contractions (Fig. 1(b)). It was very similar in all the dietary groups for the first 5 min of contractions. Beyond this time, the oxygen consumption of the $n-6$ PUFA and SF groups stabilised, whereas that of the $n-3$
PUFA group continued to increase before stabilising significantly higher after $15 \mathrm{~min}(P<0.05$ for diet; Fig. 1(b)). The decline in peak twitch tension coupled with the increased oxygen consumption led to a decrease in the efficiency index over time, independent of diet $(P<0.05$; Fig. 1(c)). The higher oxygen consumption in the $n-3$ PUFA group was associated with greater peak twitch tension in the $n$ - 3 PUFA group throughout the stimulation period and a significantly higher efficiency of oxygen utilisation $(P<0 \cdot 05$; Fig. 1(c)).

The twitch characteristics such as rise time, fall time, contraction duration and maximum rates of tension development and relaxation increased over the duration of the contraction bouts ( $P<0 \cdot 05$; Fig. 3 ). However, the $n$-3 PUFA diet attenuated the changes in twitch characteristics, resulting in significantly faster twitch development and relaxation rates and shorter rise time, fall time and twitch duration compared with either the $n-6$ PUFA or SF diet across the $30 \mathrm{~min}$ of contraction bouts $(P<0 \cdot 05)$.

\section{Blood lactate, blood glucose and muscle glycogen}

Femoral venous blood lactate concentrations increased above the resting levels $(P<0.05)$ during repetitive muscle contractions and venous $\mathrm{pH}$ decreased $(P<0.05)$ (Fig. 1(d) and (e)). Lactate concentrations increased rapidly initially (within $60 \mathrm{~s}$ ) and continued to increase gradually throughout the stimulation period. Venous lactate concentrations and $\mathrm{pH}$ changes were augmented in the $n$ - 3 PUFA group and were significantly different from those in both the $n-6$ PUFA and SF groups $(P<0 \cdot 05)$. 

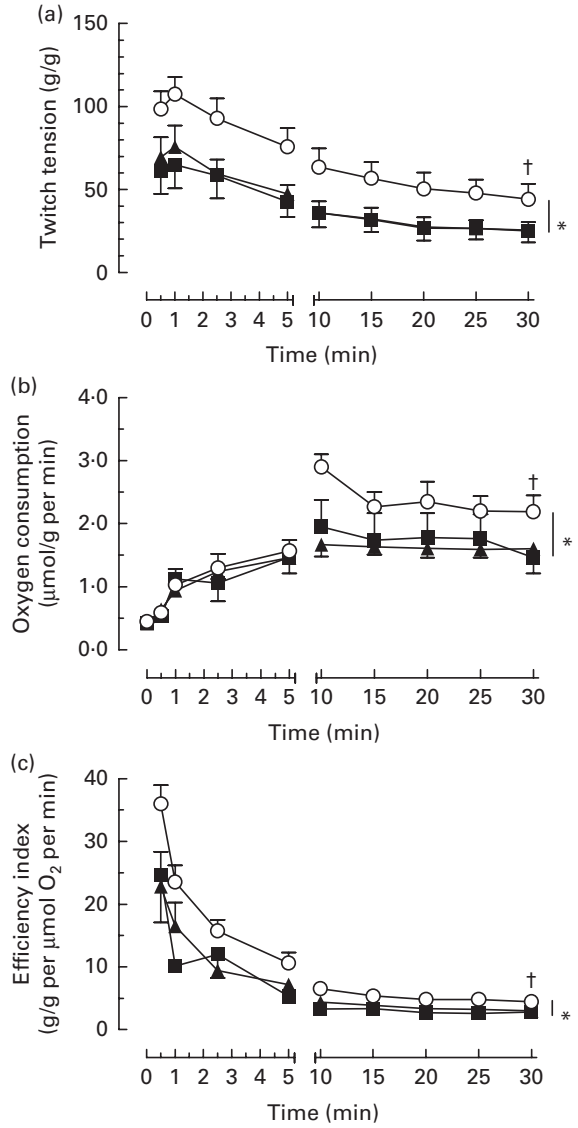

(d)
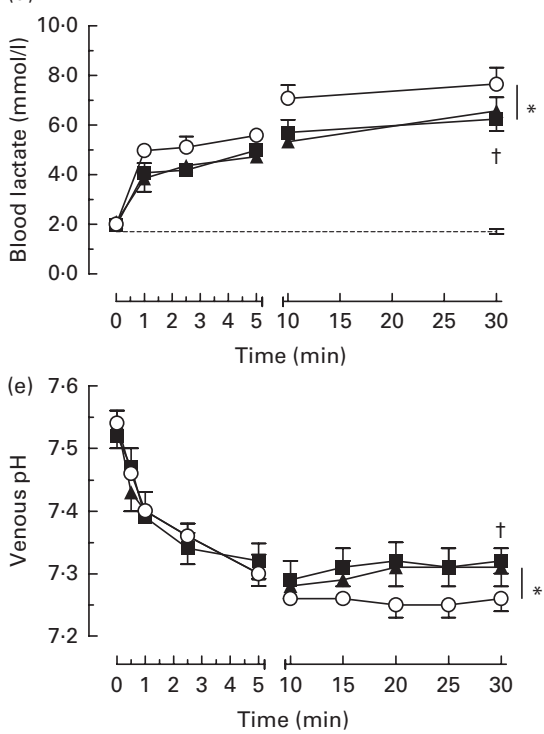

Fig. 1. Effect of dietary fat on mean contractile and blood measures over time in the gastrocnemius-plantaris-soleus muscle bundle during $30 \mathrm{~min}$ repetitive sciatic nerve stimulation bouts $(2 \mathrm{~Hz}, 7-12 \mathrm{~V}, 0.05 \mathrm{~ms})$. (a) Isometric twitch tension, (b) oxygen consumption, (c) efficiency index (tension developed per mol $\mathrm{O}_{2}$ consumed), (d) blood lactate concentrations (symbols represent femoral venous lactate concentrations and horizontal dashed line represents arterial lactate concentrations) and (e) femoral venous $\mathrm{pH}$. Time 0 represents prestimulation and is not shown for twitch tension or efficiency index. Values are means ( $n 6$ per group), with their standard errors represented by vertical bars which are omitted when smaller than the symbol. * Mean values were significantly different between the dietary groups $(P<0.05)$. † Mean values were significantly different within the dietary groups over time $(P<0.05)$.

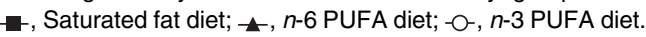

There were no differences in arterial glucose concentrations (SF: $5 \cdot 8$ (0.2 SEM) mmol/l; $n$-6 PUFA: $5 \cdot 7$ (0.3 sEM) mmol/l; $n-3$ PUFA: $6 \cdot 1(0.3 \mathrm{sEM}) \mathrm{mmol} / \mathrm{l})$ or femoral venous glucose concentrations (SF: $5.5(0.2$ SEM) mmol/l; $n-6$ PUFA: $5.5(0.3$ SEM) mmol/1; $n-3$ PUFA: $6 \cdot 0(0 \cdot 3 \mathrm{sEM}) \mathrm{mmol} / \mathrm{l})$ between the dietary groups at rest. Femoral venous glucose concentrations did not change throughout the contraction period $(30 \mathrm{~min})$ (SF: 4.8 (0.5 SEM) mmol/l; $n$-6 PUFA: 5.4 ( $0.5 \mathrm{sEM}) \mathrm{mmol} / \mathrm{l}$; $n$-3 PUFA: 5.7 (0.6 SEM) mmol/l). Glycogen concentrations in the stimulated muscle after $30 \mathrm{~min}$ of repetitive contractions were lower than those in the control muscle $(P<0.05)$, and yet they were not affected by diet in either the control gastrocnemius muscle (SF: 9.33 (1.09 SEM) mg/g wet weight; $n-6$ PUFA: $9 \cdot 19$ (0.46 SEM) mg/g wet weight; $n$-3 PUFA: 8.78 (0.70 $\mathrm{SEM}) \mathrm{mg} / \mathrm{g}$ wet weight) or the stimulated gastrocnemius muscle (SF: 6.93 (1.19 sEM) mg/g wet weight; $n$-6 PUFA: $6 \cdot 11$ (0.64 SEM) $\mathrm{mg} / \mathrm{g}$ wet weight; $n-3$ PUFA: 5.78 (0.67 SEM) $\mathrm{mg} / \mathrm{g}$ wet weight) following $30 \mathrm{~min}$ of contractions. The results obtained for glycogen were reflected in both the soleus and the plantaris muscle (data not shown).

\section{Discussion}

In rats fed fish oil, skeletal muscle was resistant to fatigue during repetitive twitch contractions in vivo, extending the original observations of the modulation of $\mathrm{VO}_{2}$ in skeletal muscle by fish oil during bouts of contraction and recovery ${ }^{(14)}$,
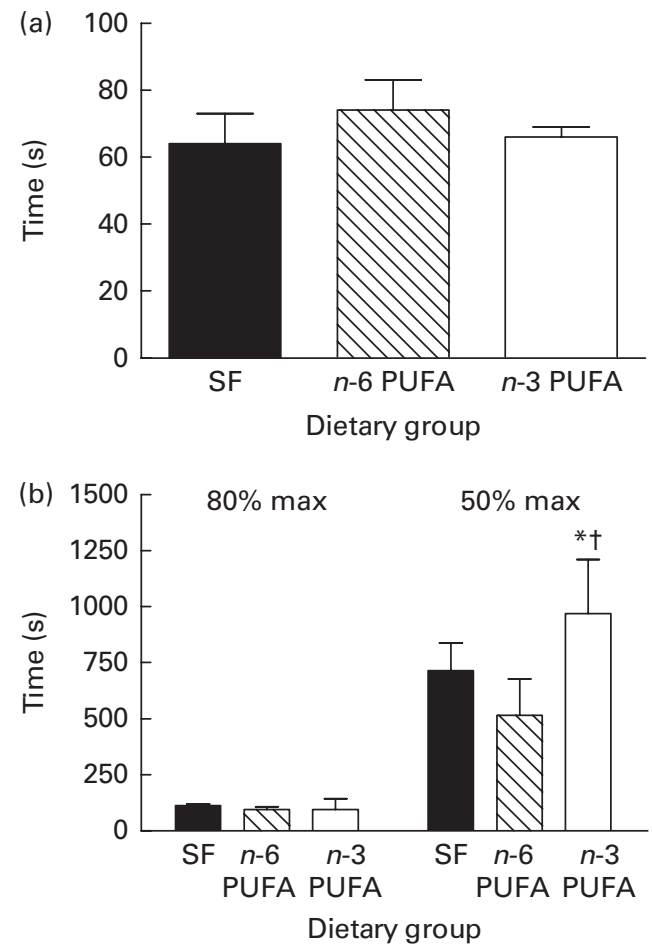

Fig. 2. Effect of dietary fat on (a) time from the first twitch to the maximum peak twitch tension, (b) time for peak twitch tension to decline to 80 and $50 \%$, respectively, of the maximum (max) peak twitch tension. Values are means ( $n 6$ per group), with their standard errors represented by vertical bars. * Mean value was significantly different from that of the $n-6$ PUFA dietary group $(P<0.05)$. † Mean value was significantly different from that of the saturated fat $(\mathrm{SF})$ dietary group $(P<0 \cdot 1)$. 
(a)

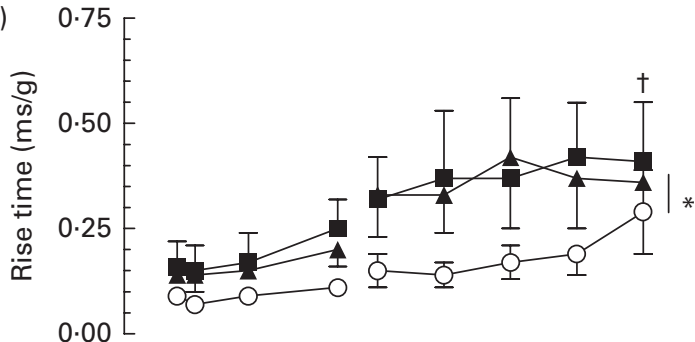

$0.00{ }^{-}$

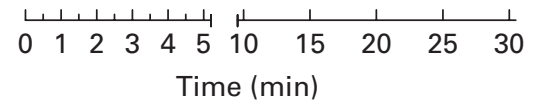

(b)

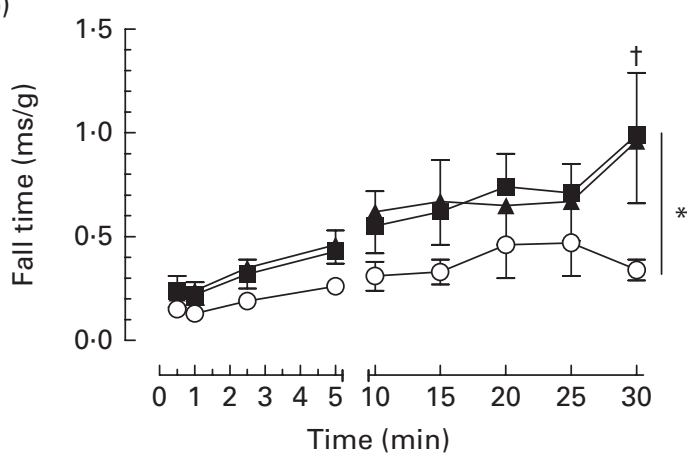

(c)

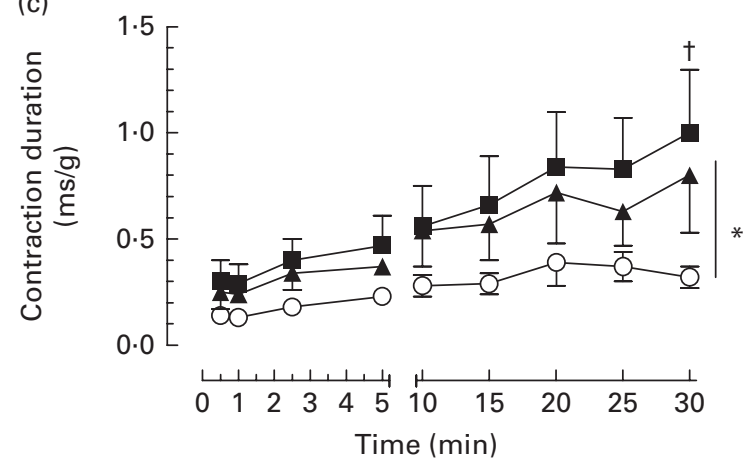

Fig. 3. Effect of dietary fat on contractile function over time in the gastrocnemius-plantaris-soleus muscle bundle during $30 \mathrm{~min}$ repetitive sciatic nerve stimulation bouts $(2 \mathrm{~Hz}, 7-12 \mathrm{~V}, 0.05 \mathrm{~ms})$. (a) Relative rise time (time to increase from 10 to $90 \%$ maximum developed tension), (b) fall time (time to decrease from 90 to $10 \%$ maximum tension) and (c) contraction duration (at $50 \%$ maximum tension). Values are means ( $n 6$ per group), with their standard errors represented by vertical bars which are omitted when smaller than the symbol. ${ }^{*}$ Mean values were significantly different between the dietary groups $(P<0.05)$. † Mean values were significantly different within the dietary groups over time $(P<0.05)$. $\mathbf{n}$, Saturated fat diet; $\mathbf{\Delta}, n-6$ PUFA diet; o, $n$-3 PUFA diet.

and in the myocardium ${ }^{(28)}$. This represents an increased efficiency of contraction-dependent oxygen use over a range of force production with no dietary differences in basal $\mathrm{VO}_{2}$, similar to that observed in the $\mathrm{K}^{+}$-arrested quiescent heart ${ }^{(28)}$.

The fatigue process could be clearly divided into an early phase of rapid decline in twitch tension achieved with consecutive contractions followed by a slow phase. In the $n-3$ PUFA rats, the early phase was characterised by greater contractile force and a reduced rate of fatigue with no extra cost of $\mathrm{VO}_{2}$ (increased $\mathrm{O}_{2}$ use efficiency). In contrast, there was little difference in efficiency in the later phase of fatigue between the dietary groups, where higher contraction force in the $n-3$ PUFA rats was matched by higher $\mathrm{VO}_{2}$ and more rapid force development and relaxation. The early rapid fatigue during low-frequency stimulation (in which oxidative metabolism predominates) $)^{(29)}$ has been attributed to metabolic changes ${ }^{(30)}$, which may in turn be attributable to the accumulation of inorganic phosphate $\left(\mathrm{P}_{\mathrm{i}}\right)$ observed in a similar preparation of hindlimb muscle used in the present study. The accumulation of $\mathrm{P}_{\mathrm{i}}$ reduces $\mathrm{Ca}^{2+}$ sensitivity and precipitates $\mathrm{CaP}_{i}$ to reduce sarcoplasmic reticulum $\mathrm{Ca}^{2+}$ release. The secondary slowing of contraction and relaxation suggests retarded removal of $\mathrm{Ca}^{2+}$ from the cytoplasm, which may be attributable to poor energy efficiency powering the sarcoplasmic reticulum ATPase or inhibition by $\mathrm{P}_{\mathrm{i}}$ or reactive oxygen species ${ }^{(31)}$. Reduced rates of calcium release from the sarcoplasmic reticulum into the cytosol and re-uptake by the sarcoplasmic reticulum $\mathrm{Ca}^{2+}$-ATPase are implicated in muscle fatigue ${ }^{(32)}$, and in the present study, DHA protection against the fatigue-related slowing of relaxation time in turn implicates attenuation of the impairment of cytoplasmic $\mathrm{Ca}^{2+}$ re-uptake ${ }^{(33)}$. There is a parallel with the altered cellular $\mathrm{Ca}^{2+}$ handling implicated in many of the effects of dietary fish oil in the heart ${ }^{(34)}$, including the modulation of excessive oxygen consumption and cardiac arrhythmias, both of which are usually associated with conditions of cellular sarcoplasmic reticulum and mitochondrial $\mathrm{Ca}^{2+}$ overload ${ }^{(28,35)}$. The increased efficiency has a further parallel to the reduced whole-body $\mathrm{VO}_{2}$ throughout the prolonged exercise bouts in trained cyclists supplemented with fish oil ${ }^{(17)}$ and improved post-ischaemic cardiac recovery in rats ${ }^{(28,36)}$.

The optimal oxygenation and perfusion properties of the in vivo autologous hindlimb model ${ }^{(23)}$, adapted from canine muscle fatigue experiments ${ }^{(37)}$, were important contributors to the observations made in the present study. Isolated muscle preparations are often compromised by non-physiological operating temperatures or by oxygen delivery impaired by low oxygen-carrying capacity of crystalline perfusate and limited perfusion into large muscle bundles in vitro ${ }^{(38)}$ and by abnormal accumulation of metabolites and electrolytes in the absence of perfusion. These methodological deficiencies could, therefore, explain the failure of an earlier study to observe the effects of dietary LC $n-3$ PUFA-induced changes in membrane composition on skeletal muscle function in vitro, except in relation to an essential fatty acid-deficient diet $^{(13)}$

Fish oil feeding leads to the incorporation of LC $n$-3 PUFA DHA into the hindlimb skeletal muscle ${ }^{(14)}$. This is the principal n-3 PUFA incorporated into excitable tissues, including heart and skeletal muscles in humans and animals ${ }^{(34)}$, even when high-EPA fish oil or purified EPA is consumed ${ }^{(7,9,39)}$. The similarities between the effects of fish oil on skeletal muscle and myocardial function and fatty acid composition suggest common underlying mechanisms. In the present study, the rise time and fall time of force production were found to be not slowed in the muscle of the $n$ - 3 PUFA group to the same extent as in that of the SF and $n-6$ PUFA groups. Therefore, the $\mathrm{Ca}^{2+}$ cycling process appears to be one potential mechanism of action of fish oil fatty acids in the function of 
excitable cells that may translate to skeletal muscle. Interestingly, the higher unsaturation index of skeletal muscle membrane phospholipids derived from dietary fish oil implies an increased risk of cellular oxidative damage, which might be expected to translate into poor contractile performance in line with the free-radical theory of fatigue ${ }^{(40)}$. However, similar to exercise training, which puts muscles under increased oxidative stress, yet preconditions against subsequent stressinduced cellular damage, the paradoxical fatigue resistance provoked by dietary fish oil suggests that similar to ischaemic preconditioning $^{(41)}$ and nutritional preconditioning by LC $n-3$ PUFA in the heart ${ }^{(36)}$ or exercise training ${ }^{(42)}$, the LC $n-3$ PUFA up-regulate endogenous protective mechanisms in skeletal muscle. However, future research will need to specifically address these aspects.

There was no evidence in the present study that dietinduced differences in substrate accumulation consistent with vigorous exercise were responsible for the observed contractile changes. Lactate production increased as muscles fatigued; however, as the venous concentrations were greatest in the $n-3$ PUFA tissues that fatigued least, these results support the assertion that lactate and hydrogen ion $\left(\mathrm{H}^{+}\right)$ accumulation is not a major cause of fatigue ${ }^{(38)}$. Rather, lactate production, and subsequent decrease in $\mathrm{pH}$, simply reflects the sustained contractile function of muscles and a greater extent of anaerobic metabolism ${ }^{(43)}$. In turn, such changes to local metabolic conditions can enhance oxygen extraction as observed in fish oil-fed rats during the later phase of fatigue through a rightward shift in the oxygen $-\mathrm{Hb}$ dissociation curve, permitting greater oxygen unloading in the $n-3$ PUFA skeletal muscle, despite provision of a constant blood flow and arterial $\mathrm{PO}_{2}$. These results also further reflect the tight coupling of oxygen supply and demand where the efficient use of oxygen, in muscles with high DHA concentrations, will ultimately result in more prolonged and sustained force development.

A number of further questions can be explored with regard to the role of DHA in skeletal muscle membranes and fatigue. To date, studies on muscle contraction have been limited to twitch conditions. Although this is important for understanding a basic level of interaction between DHA and muscle function, there is further need to investigate the role of fish oil under physiologically relevant conditions of intermittent tetanic muscle contractions that demand a greater turnover of intracellular calcium. It is also intriguing to postulate that possible dietary fish oil preconditioning in skeletal muscle may allow preparation for stressful conditions where oxygen delivery may be depressed, such as hypoxia or peripheral ischaemia. Equally, given that epidemiological data now support the role of LC $n$-3 PUFA in the diet with respect to indices of maintained muscle function ${ }^{(44)}$, there is good reason to further explore the potential for enhanced muscle physiology or simply attenuated decline by increasing DHA content in human diets. Most importantly, 'low-dose' fish oil, independent of background n-6 PUFA concentrations, can achieve equivalent membrane DHA incorporation in skeletal muscle ${ }^{(45)}$, and this concentration is more than achievable in human diets (one to two meals of fatty fish per week).
In summary, using the rat in vivo autologous pumpperfused hindlimb model, in the present study, it was found that muscles sustained higher repeated contractile force after fish oil supplementation, which was objectively measured as an increased time to fatigue. Modulation of skeletal muscle oxygen consumption was evident across multiple time points through the fatigue process, associated with increased membrane DHA concentrations. With the exclusion of cardiac and vascular mechanisms that affect oxygen delivery, the increased oxygen efficiency coupled with attenuation of the fatigue-related prolongation of twitch duration and slowed recovery to baseline from peak twitch force points towards improved handling of calcium in the skeletal muscle cells. The present findings demonstrate that DHA is incorporated into skeletal muscle membranes to provide direct nutritional preconditioning to sustain muscle contractile performance. Preconditioning and improved muscle function have traditionally been associated with exercise training stimulus. However, in populations with skeletal muscle dysfunction precluding exercise training, such as the frail and elderly, the revelation of these potential benefits of dietary LC $n-3$ PUFA is exciting and points to future human applications.

\section{Acknowledgements}

The high-DHA fish oil was provided by Nu-Mega Ingredients.

The present study was jointly supported by an Australian Research Council - Strategic Partnerships with Industry Research and Training (ARC-SPIRT) grant with Clover Corporation, Sydney, Australia. G. E. P. was supported by the ARC Key Centre for Smart Foods (University of Wollongong). None of the organisations had any role in the design and data analysis of the study or in the writing of this article.

The authors' contributions were as follows: G. E. P. and P. McL. contributed equally to all aspects of manuscript preparation.

None of the authors has any conflicts of interest.

\section{References}

1. Barclay JK (1993) A delivery-independent blood flow effect on skeletal muscle fatigue. J Appl Physiol 74, 2294-2300.

2. Gladden LB, McIntosh BR \& Stainsby WN (1978) Oxygen uptake and developed tension during and after fatigue, curare block, and ischemia. J Appl Physiol 45, 751-756.

3. Gorman MW, Barclay JK \& Sparks HV (1988) Effects of ischemia on $\mathrm{VO}_{2}$ tension and vascular resistance in contracting canine skeletal muscle. J Appl Physiol 65, 1075-1081.

4. Hogan MC, Arthur PG, Bebout PD, et al. (1992) Role of $\mathrm{O}_{2}$ in regulating tissue respiration in dog muscle working in situ. J Appl Physiol 73, 728-736.

5. Hogan MC, Kurdak SS \& Arthur PG (1996) Effect of gradual reduction in $\mathrm{O}_{2}$ delivery on intracellular homeostasis in contracting skeletal muscle. J Appl Physiol 80, 1313-1321.

6. Gorjao R, Azevedo-Martins A, Rodrigues H, et al. (2009) Comparative effects of DHA and EPA on cell function. Pharmacol Ther 122, 56-64.

7. McLennan P, Howe P, Abeywardena M, et al. (1996) The cardiovascular protective role of docosahexaenoic acid. Eur J Pharmacol 300, 83-89. 
8. McLennan P, Abeywardena MY \& Charnock JS (1988) Dietary fish oil prevents ventricular fibrillation following coronary artery occlusion and reperfusion. Am Heart J 116, 709-717.

9. Pepe S \& McLennan PL (1996) Dietary fish oil confers direct antiarrhythmic properties on the myocardium of rats. $J$ Nutr 126, 34-42.

10. Charnock JS, Abeywardena MY, Poletti VM, et al. (1992) Differences in fatty acid composition of various tissues of the marmoset monkey after different lipids supplement diets. Comp Biochem Physiol 101A, 387-393.

11. McLennan PL, Bridle TM \& Abeywardena MY (1992) Dietary lipid modulation of ventricular fibrillation threshold in the marmoset monkey. Am Heart J 123, 1555-1561.

12. Pan D \& Storlien LH (1993) Dietary lipid profile is a determinate of tissue phospholipids fatty acid composition and rate weight gain in rats. $J$ Nutr 123, 512-519.

13. Ayre K \& Hulbert A (1996) Dietary fatty acid profile influences the composition of skeletal muscle phospholipids in rats. J Nutr 126, 653-662.

14. Peoples G \& McLennan P (2010) Dietary fish oil reduces skeletal muscle oxygen consumption, provides fatigue resistance and improves contractile recovery in the rat in vivo hindlimb. BrJ Nutr 104, 1771-1779.

15. Clavel S, Farout L, Briand M, et al. (2002) Effect of endurance training and/or fish oil supplemented diet on cytoplasmic fatty acid binding protein in rat skeletal muscles and heart. Eur J Appl Physiol 87, 193-201.

16. Kamolrat T, Gray S \& Thivierge M (2012) Fish oil positively regulates anabolic signalling alongside an increase in wholebody gluconeogenesis in ageing skeletal muscle. Eur $J$ Nutr 52, 647-657.

17. Peoples G, McLennan P, Howe P, et al. (2008) Fish oil reduces heart rate and oxygen consumption during exercise. J Cardiovasc Pharmacol 52, 540-547.

18. Peoples G, Hoy A, Henry R, et al. (2013) Autologous pumpperfused rat hindlimb preparation for investigating muscle function and metabolism in vivo. Microcirculation 20, 511-523.

19. Buckley J, Burgess S, Murphy K, et al. (2009) DHA-rich fish oil lowers heart rate during submaximal exercise in elite Australian Rules footballers. J Sci Med Sport 12, 503-507.

20. Oostenburg GS, Mensink RP, Hardeman MR, et al. (1997) Exercise performance, red blood cell deformability, and lipid peroxidation: effects of fish oil and vitamin E. $J A p p l$ Physiol 83, 746-752.

21. Raastad T, Hostmark AT \& Stromme SB (1997) Omega-3 fatty acid supplementation does not improve maximal aerobic power, anaerobic threshold and running performance in well trained soccer players. Scand J Med Sci Sports 7, 25-31.

22. Mickleborough $\mathrm{T}$ (2013) Omega-3 polyunsaturated fatty acids in physical performance optimisation. Int J Sport Nutr Exerc Metab 23, 83-96.

23. Hoy A, Peoples G \& McLennan P (2009) The effect of vasoconstrictors on oxygen consumption in resting and contracting skeletal muscle of the autologous pump-perfused rate hindlimb. J Physiol Pharmacol 60, 155-160.

24. Reeves P, Nielsen F \& Fahey G (1993) AIN-93 purified diets for laboratory rodents: final report of the American Institute of Nutrition ad hoc writing committee on the reformulation of the AIN-76A rodent diet. J Nutr 123, 1939-1951.

25. Owen A, Peter-Przyborowska B, Hoy A, et al. (2004) Dietary fish oil dose- and time-response effects on cardiac phospholipid fatty acid composition. Lipids 39, 955-961.
26. Hogan MC, Gladden LB, Grassi B, et al. (1998) Bioenergetics of contracting skeletal muscle after partial reduction of blood flow. J Appl Physiol 84, 1882-1888.

27. Stainsby WN \& Renkin EM (1961) Autoregulation of blood flow in resting skeletal muscle. Am J Physiol 201, 117-122.

28. Pepe S \& McLennan PL (2002) Cardiac membrane fatty acid composition modulates myocardial oxygen consumption and postischemic recovery of contractile function. Circulation 105, 2303-2308.

29. Fitts RH (1994) Cellular mechanisms of muscle fatigue. Physiol Rev 74, 49-74.

30. Baker A, Carson P, Miller R, et al. (1994) Metabolic and non-metabolic components of fatigue monitored by ${ }^{31}$ P-NMR. Muscle Nerve 17, 1002-1009.

31. Allen D, Lamb G \& Westerblab H (2008) Skeletal muscle fatigue: cellular mechanisms. Physiol Rev 88, 287-332.

32. Favero TG (1999) Sarcoplasmic reticulum $\mathrm{Ca}^{2+}$ release and muscle fatigue. J Appl Physiol 87, 471-483.

33. Verberg E, Thorud H, Eriksen M, et al. (2001) Muscle contractile properties during intermittent nontetanic stimulation in rat skeletal muscle. Am J Physiol Regul Integr Comp Physiol 281, R1952-R1965.

34. McLennan P \& Abeywardena MY (2005) Membrane basis for fish oil effects on the heart: linking natural hibernators to prevention of human sudden cardiac death. J Membr Biol 206, 85-102.

35. Pepe S, Tsuchiya N, Lakatta EG, et al. (1999) PUFA and aging modulate cardiac mitochondrial membrane lipid composition and $\mathrm{Ca}^{2+}$ activation of PDH. Am J Physiol 276, H149-H158.

36. Abdukeyum G, Owen A \& McLennan P (2008) Dietary (n-3) long chain polyunsaturated fatty acids inhibit ischemia and reperfusion arrhythmias and infarction in rat heart not enhanced by ischemic preconditioning. $J$ Nutr 138, 1902-1909.

37. Stainsby WN \& Welch HG (1966) Lactate metabolism of contracting dog skeletal muscle in situ. Am J Physiol 211, $177-183$.

38. Zhang S, Bruton J, Katz A, et al. (2006) Limited oxygen diffusion accelerates fatigue development in mouse skeletal muscle. J Physiol 572, 551-559.

39. Harris W, Sands S, Windsor S, et al. (2004) Omega-3 fatty acids in cardiac biopsies from heart transplantation patients - correlation with erythrocytes and response to supplementation. Circulation 110, 1645-1649.

40. Ferreira L \& Reid M (2008) Muscle-derived ROS and thiol regulation in muscle fatigue. $J$ Appl Physiol 104, 853-860.

41. al Makdessi S, Brandle M, Ehrt M, et al. (1995) Myocardial protection by ischemic preconditioning: the influence of the composition of myocardial phospholipids. Mol Cell Biochem 145, 69-73.

42. Pansarasa O, D'Antona G, Gualea M, et al. (2002) "Oxidative stress": effects of mild endurance training and testosterone treatment on rat gastrocnemius muscle. Eur J Appl Physiol 87, 550-555.

43. Westerblab H, Bruton J \& Katz A (2010) Skeletal muscle: energy metabolism, fibre types, fatigue and adaptability. Exp Cell Res 316, 3093-3099.

44. Abbatecola A, Cherubini A, Guralnik J, et al. (2009) Plasma polyunsaturated fatty acids and age-related physical performance decline. Rejuvenation Res 12, 25-32.

45. Slee E, McLennan P, Owen A, et al. (2010) Low dietary fish-oil threshold for myocardial membrane n-3 PUFA enrichment independent of n-6 PUFA intake in rats. J Lipid Res 51, 1841-1848. 